\title{
Е.В. ДОВБНЯ
}

\section{Проблемы тематического поиска в электронном каталоге научной библиотеки: обзор исследований}

\begin{abstract}
Реферат. Научные библиотеки используют разные подходы и средства тематического поиска. Вопросы, связанные с электронным тематическим поиском, широко обсуждаются в библиотечном сообществе. Целью данного обзора является анализ публикаций последних лет, посвященных аспектам и проблемам, связанным с тематическим поиском в электронном каталоге (ЭК). При его подготовке использованы публикации (и библиография к ним), взятые из национальной библиографической базы данных - Российского индекса научного цитирования (РИНЦ), временной охват - 2003-2019 годы. Особое внимание в исследованиях уделяется необходимости сочетания разных информационно-поисковых языков (ИПЯ), тезаурусов, предметных рубрик, ключевых слов для обеспечения эффективного и многоаспектного поиска. Так как в отечественных библиотеках накоплен большой опыт по индексированию и поиску посредством иерархических классификаций (УДК и ББК), необходимо наиболее полно использовать это в электронном тематическом поиске. Авторам последних исследований, касающихся тематического поиска, наиболее эффективным представляется функционирование классификационных систем в составе комплекса лингвистических средств ЭК. В электронной среде становится возможным использовать классификационные индексы как совокупность знаков, максимально отражающих содержание документа, что предполагает использование всех фрагментов индекса как независимых поисковых элементов. Однако для эффективного использования в ЭК требуется модификация лексического состава классификационных ИПЯ. Основными направлениями здесь могут стать: унификация структуры и лексики классификационных таблиц, оптимизация структуры систем классификации (с использованием принципов фасетизации), а также формализация использования грамматических средств (это необходимо для обеспечения однозначности их интерпретации в ЭК). В обзоре представлены и проанализированы направления, по которым развивается тематический поиск в электронной среде. Например, разработана и внедряется надстройка над УДК (БЕН РАН), что позволяет пользователям библиотеки в удаленном доступе вести полноценный тематический поиск. Представлены также новые разработки Российской государственной библиотеки по тематическому поиску, в частности, проект «Представление классификационных метаданных электронных библиотек по технологии связанных данных». Делается вывод о необходимости формирования общего пространства связанных открытых данных различных библиотек.

Ключевые слова: библиотечно-информационное обслуживание, тематический поиск, электронный каталог, иерархические классификации, предметное индексирование, информационно-поисковые языки, ББК, УДК, научные библиотеки.

Для цитирования: Довбня Е.В. Проблемы тематического поиска в электронном каталоге научной библиотеки: обзор исследований // Библиотековедение. 2020. Т. 69, № 4. С. 367-374. DOI: 10.25281/0869-608X-2020-69-4-367-374.

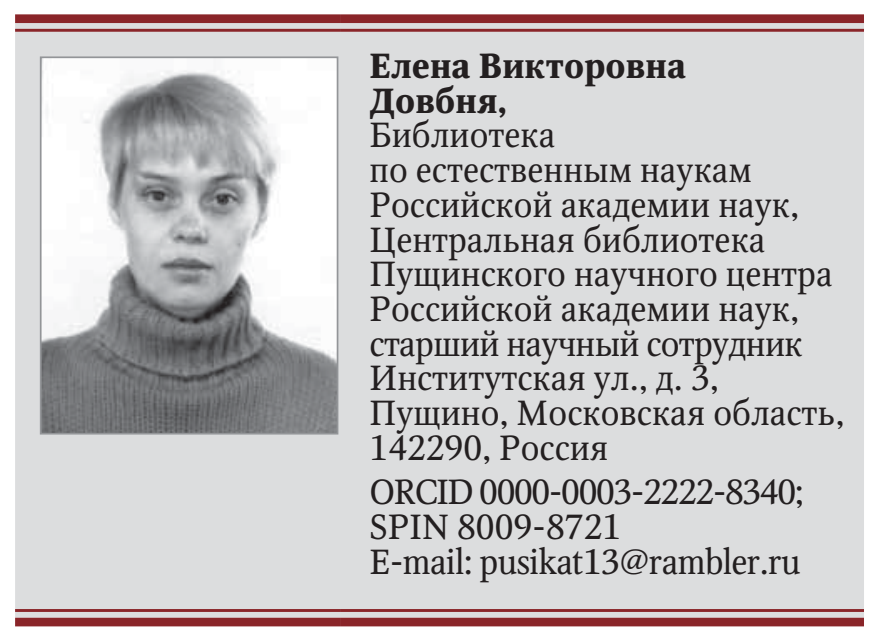


Э лектронный тематический поиск, подходы и средства, применяемые научными библиотеками для этого поиска, - уже давно одна из самых обсуждаемых тем в библиотечном деле. Российские библиотеки используют в электронном тематическом поиске разработки, позволяющие сочетать различные информационно-поисковые языки, ключевые слова, тезаурусы, предметные рубрики, а также языки иерархических классификаций (УДК и ББК).

Цель настоящей работы - анализ публикаций последних лет, посвященных аспектам и проблемам, связанным с тематическим поиском в электронном каталоге (ЭК). При подготовке обзора были использованы публикации (и библиография к ним) из национальной библиографической базы данных - Российского индекса научного цитирования (РИНЦ), временной охват - 2003-2019 годы.

Очень емко и точно о сути поиска, в частности о тематическом поиске, высказался Э.Р. Сукиасян: «Поиск - естественный процесс для любого человека, относящего себя к homo sapiens, человека, стремящегося понять окружающий мир. Поскольку эвристическая поисковая активность всегда сопровождает когнитивное развитие личности, человек может быть назван homo quaerens - человек ищущий» [1, с. 74].

Предметом тематического поиска выступает «тема документа, его составной части, фрагмента (включая персональный, географический и др. аспекты), выраженная в классификационных индексах, предметных рубриках, ключевых словах в библиографической записи, наименованиях разделов и рубрик библиографических пособий» [2, с. 49].

Основной проблемой, как показывает опыт последних лет, является ошибочное мнение о том, что в ЭК библиотек можно вести тематический поиск так же, как это было в карточном систематическом каталоге. Действительно, современные ЭК обеспечивают пользователю новые, ранее недоступные возможности. Но это касается, как правило, поиска по заглавию, автору, редактору, составителю, коллективному автору, слову из заглавия и т. д. В случае же тематического поиска все оказывается намного сложнее. В поисковых инструментах многих ЭК отсутствует основной принцип построения систематического каталога - его иерархическая структура, соподчинение одних понятий другим, поиск по принципу «вверх - вниз», от широкотематического к узкотематическому запросу и наоборот. В результате поиск становится малоэффективным, происходят большие потери информации.

О наиболее актуальных вопросах и проблемах, связанных с тематическим поиском в ЭК современной научной библиотеки, за последние годы писали такие известные авторы (в том числе в соавторстве): О.А. Лавренова [3], Г.А. Скарук [4], 3.М. Миниярова [5; 6], С.А. Власова [7; 8], А.А. Ивановский [9; 10], Н.Е. Каленов [11; 12], А.Н. Сысоев $[13 ; 14]$ и многие другие.

Библиотека, чтобы выбрать наиболее эффективные средства тематического поиска, прежде всего должна решить, каковы информационные потребности (поисковые намерения) ее пользователей. Как правило, выделяют следующие типы поисковых намерений:

«...1) найти хоть что-то, но точно по предмету поиска;

2) подобрать максимально полную информацию по теме, но желательно с минимальным показателем “информационного шума” (некоторые потери допустимы);

3) найти все, что касается темы поиска (пусть попадутся лишние документы, но не упустить что-то новое и интересное);

4) сначала определиться, какие существуют темы в интересующей области знания» [3, c. 44].

В связи с этим, очевидно, встает вопрос о комплексном использовании всех информационно-поисковых языков (ИПЯ) - вербальных (языки библиографического описания, предметных рубрик и ключевых слов) и классификационных (языки иерархических классификаций, используемых в библиотеках).

Об актуальных проблемах интеллектуального доступа в обзорной статье пишет Н.И. Тютчева: «Чаще всего используются индексы иерархических классификаций в режиме предкоординации и язык предметных рубрик - в режиме посткоординации (иерархические классификации, язык предметных рубрик, язык ключевых слов, слова из заглавий, дескрипторный информационно-поисковый язык) - все информационно-поисковые языки» [15].

Масштабное исследование, посвященное использованию различных средств тематического поиска в работе крупных библиотек, было выполнено в Государственной публичной научно-технической библиотеке (ГПНТБ) Си- 
бирского отделения Российской академии наук (СО РАН) [4]. Его результаты дают представление о том, какие ИПЯ используются в процессе тематического поиска. Так, Российская государственная библиотека (РГБ) использует в электронном поиске иерархические классификации (ИК), язык предметных рубрик (ЯПР), язык ключевых слов (ЯКС), слова из заглавия документа (С3); Российская национальная библиотека - ИК, ЯПР, ЯКС; ГПНТБ России ЯПР, ЯКС, СЗ; ГПНТБ СО РАН - ИК, ЯПР, ЯКС, СЗ; Библиотека по естественным наукам (БЕН) РАН - ИК, СЗ; Всероссийская государственная библиотека иностранной литературы им. М.И. Рудомино - ЯПР; Фундаментальная библиотека Санкт-Петербургского государственного политехнического университета ИК, ЯПР; Научная библиотека Новосибирского государственного технического университета ЯПР, дескрипторный информационно-поисковый язык (ДИПЯ), ЯКС, СЗ [4, с. 17].

На основе этого можно сделать вывод о том, что устойчивой тенденцией является «применение в ЭК комплекса различных по структуре, лексическому наполнению и функциональным возможностям ИПЯ» [4, с. 15].

Одним из ИПЯ в ЭК ГПНТБ СО РАН стала Библиотечно-библиографическая классификация (ББК). В работах, посвященных тематическому поиску, Г.А. Скарук описывает способность иерархических классификаций к автоматизированному поиску [16; 17]. О необходимости поиска по классификационным индексам, а также их отдельным элементам автор пишет: «Возможности иерархических классификаций связываются с функцией своего рода “карты знаний”, обеспечивающей определенную целостность и обозримость представления знаний и ориентации пользователя на стадии составления поискового предписания» [16, с. 268-269].

Об уникальных поисковых возможностях классификационных ИПЯ говорится в статье «Возможности поиска по классификационным индексам в электронном каталоге» [17]. Автор считает наиболее эффективным функционирование классификационных систем в составе комплекса лингвистических средств ЭК. «Основные направления преобразований: унификация структуры и лексики классификационных таблиц, оптимизация структуры систем классификации с использованием принципов фасетизации, формализация использования грамматических средств с целью обеспечения однозначности их интерпретации в ЭК, существенное расширение ссылочно-справочного аппарата» [17, с. 28$]$.

3.М. Миниярова приводит интересные данные и исследования, касающиеся подходов к индексированию в ЭК [5; 6]. В статье «Подходы к индексированию в электронных каталогах. Возможности поэлементного поиска» говорится о целом комплексе проблем, связанных с использованием различных ИПЯ в ЭК [5]. Автор указывает: «ББК является комбинационной (иначе говорят “полуфасетной”) классификационной системой. Классификационный индекс строится из структурных элементов, в совокупности адекватно и многоаспектно раскрывающих содержание документа. Классификационный индекс ББК может практически адекватно отразить содержание документа, раскрыв до 25-30 поисковых признаков» [5, с. 26-28].

Интересный опыт в реализации тематического поиска в ЭК накоплен специалистами БЕН РАН (в том числе в соавторстве): С.А. Власовой [7; 8], Н.Е. Каленовым [11; 12], А.А. Ивановским [9; 10], А.Н. Сысоевым [13; 14].

Так как все издания, поступающие в БЕН РАН, индексируются по таблицам УДК, для тематического поиска была разработана специальная иерархическая надстройка над УдК. Подробно этот сложный проект описан в статье С.А. Власовой «Реализация тематического поиска в электронном каталоге Библиотеки по естественным наукам Российской академии наук» [7]. «Для подключения к электронному каталогу такого тематического рубрикатора была разработана специальная система создания базы данных (БД) тематических рубрик. $<$...> Пользователю предоставляется список названий рубрик верхнего уровня. Названия рубрик, которые являются активными ссылками, содержат подрубрики. Переход по выбранной ссылке позволит увидеть соответствующий список подрубрик (с указанием индексов УДК) выбранной рубрики. После завершения создания базы данных тематических рубрик, она была подключена к электронному каталогу книг и продолжающихся изданий» [7, с. 146-148]. Автор отмечает: «Специальная программа... накапливает данные поисковых запросов в SQL-таблице... Таблица содержит следующую информацию: выбранные поисковые поля и значения поисковых терминов; логические связки в запросе; дата запроса; библиотека, по фондам которой проводился поиск... количество найденных записей» [7, с. 148]. 
А.А. Ивановский в статье «Возможности поиска по классификационным индексам в электронном каталоге: опыт Библиотеки по естественным наукам Российской академии наук» описывает трудности, с которыми столкнулись разработчики иерархической надстройки над УдК: «Первая проблема - иерархия знаний в УДК отличается от общепринятой иерархии научных отраслей. Это приводит к тому, что при строгом соблюдении УдК не удается построить интуитивно понятную классификацию даже на верхних уровнях иерархии. <..> ...При построении каталога иерархия УДК нарушается. При этом происходит приближение структуры каталога к общепринятой (ожидаемой пользователем) структуре научных знаний. <...> Вторая проблема построения систематического каталога со строгим соблюдением канонов УДК заключается в отсутствии в УДК подклассов "общие вопросы”... <...> ...В систематическом каталоге, чтобы свернуть все эти темы в один пункт - “общие вопросы” какой-либо науки, приходится конструировать искусственные поисковые образы...» [9, с. 65-67].

Уникальность данной разработки состоит в том, что имеет место реализация поисковой системы в ЭК, что обеспечивает полноту результатов поиска и избавляет пользователя от трудностей с использованием УДК. Модуль «Тематический поиск» находится в свободном доступе на сайте БЕН РАН как часть ЭК книг и продолжающихся изданий.

Однако, следует отметить, что механизм поиска с помощью надстройки над УдК имеет узкую направленность. Очевидно, что, если библиографическая запись (Б3) не будет иметь индексов УДК, она не сможет участвовать в поиске. Это важно, если учесть, что далеко не все библиотеки, входящие в сеть БЕН РАН, пользуются УДК.

О.А. Лавренова подробно раскрывает современное состояние и различные аспекты тематического поиска в РГБ [18; 19]. Она отмечает, что ЭК РГБ включает в себя индексы ББК, а также ИПЯ на основе таблиц ББК с предметным входом. Таким образом, программное обеспечение ЭК РГБ позволяет «ведение поиска с верхнего или любого уровня до любого другого уровня иерархии вниз; выход на любой уровень иерархии и на уровни в разных тематических разделах одновременно по "предметному входу”; развертывание наименования делений на экране (с индексами или без них); переход от деления ББК к списку БЗ без привлечения индекса для поиска» [18, с. 34].
В статье «Тематический поиск в электронных библиотеках: иллюзии и реальность» говорится: «Наиболее распространенные ИПЯ предоставляют следующие средства тематического поиска в электронных каталогах: свободные ключевые слова (КС), перечни КС, тезаурусы, предметные рубрики (предметные классификации), иерархические классификации (индексы, наименования делений), рубрикаторы (коды, наименования рубрик - для приблизительного распределения документов по широким темам). <...> Исследование показало, что без ввода в Б3 словесных формулировок индексов и без тезаурусов при поиске в электронных каталогах получаются огромные потери информации...» $[19$, c. $3-4]$.

Особо следует отметить, что в течение 2015-2019 гг. в РГБ проводилось уникальное исследование, отраженное в последних статьях О.А. Лавреновой [20; 21]. Так, в работе «Тематический поиск электронных ресурсов на основе классификационной модели организации знаний» указано: «В последнее десятилетие наметилась тенденция вывода процессов поиска электронных ресурсов библиотек в открытое сетевое пространство по технологиям Linked Open Data (LOD, связанных открытых данных). При этом системы организации знаний публикуются библиотеками в виде словарей связанных данных (Linked Data vocabularies) в Семантической паутине (Semantic Web, Семантическом вебе)... специалисты РГБ ведут проект “Представление классификационных метаданных электронных библиотек по технологии связанных данных (Linked Open Data)...”. Он поддержан грантом РФФИ № 15-07-05265. <...> Задача публикации в LOD систем организации знаний, в частности классификаций, заключается в первую очередь в формировании отдельных утверждений, построенных в среде описания ресурсов RDF (Resource Description Framework). Pecypсами считаются любые данные, в том числе элементы классификации. Каждый ресурс получает URI (Uniform Resource Identifier - универсальный идентификатор ресурса в Сети), т. е. уникальный адрес. <...> В технологии LOD требуется обеспечить процессы обогащения запроса поисковыми признаками исключительно с помощью программных средств (без участия человека). Каждый классификационный индекс объявляется концептом и получает URI. B форме триплетов (субъект предикат - объект) представляются все связи 
индекса с теми элементами классификации, которые могут использоваться для программного обогащения запроса человека. Таковыми считаются эквиваленты слов из формулировок (грамматические формы, результаты словообразования, синонимы и т. д.), иерархические и ассоциативные связи между индексами, ассоциативные связи (ссылки) с другими ресурсами в LOD. <...> Это позволяет связать в Ceмантической паутине что угодно с чем угодно, а также обеспечить поиск открытых связанных данных стандартными программными средствами с обогащением запросов на основе зафиксированных связей» [20, с. 60-61].

В докладе на Международной научнопрактической конференции «Румянцевские чтения - 2019» О.А. Лавренова, подводя предварительные итоги проекта РГБ, делает следующие выводы: «Представляется целесообразным сформировать некоторое общее семантическое пространство для библиотек. В качестве основы предлагается Классификационная система организации знаний, созданная в РГБ на базе полного варианта ББК и собственного Генерального систематического каталога (ГСК)... Система опубликована в среде связанных открытых данных (Linked Open Data, LOD)... под открытой лицензией РГБ... <..> Классификации, файлы предметных рубрик, тезаурусы, структурированные в цифровой форме, требуется представить в среде LOD с использованием RDF-схемы и пространства имен SKOS. Вначале планируется поработать над технологиями установления соответствий данных в Классификационной системе со средним вариантом таблиц ББК, таблицами УДК, тезаурусом по медицине MESH, тезаурусом-справочником географических названий РГБ. Обогащение запросов на основе такого рода связей должно обеспечить в дальнейшем возможности проведения поиска по этим ресурсам из одной точки на основе Классификационной системы» [21, с. 105-109].

В заключение отметим, что тематический поиск в ЭК и электронных библиотеках постоянно трансформируется в соответствии с развитием новых технологий. Основной идеей последних исследований в этой области является идея объединения технологий тематического поиска в различных библиотеках. То, что это становится возможным, наглядно показывают последние итоги разработок РГБ. Очевидно, что такие масштабные задачи невозможно осуществить без соответствующего финансирования.

\section{Список источников}

1. Сукиасян Э.P. Homo Quaerens (Человек ищущий). К проблеме развития познавательных способностей читателя в процессе поиска // Научные и технические библиотеки. 2002. № 4. С. 73-78.

2. Левин Г.Л. Библиографический поиск: теоретический аспект // Библиотековедение. 2000. № 4. C. 47-50.

3. Лавренова О.А. Тематический поиск в электронных каталогах и электронных библиотеках // Библиотековедение. 2004. № 5. С. 42-50.

4. Скарук Г.А., Жарикова Л.А., Стукалова А.А. Поисковые языки электронных каталогов : конспект лекции по курсу «Справочно-поисковый аппарат». Новосибирск : ГПНТБ СО РАН, 2009. 44 с.

5. Миниярова 3.М. Подходы к индексированию в электронных каталогах. Возможности поэлементного поиска // Научные и технические библиотеки. 2005. № 12. С. 25-35.

6. Миниярова 3.М. Электронный каталог - особая поисковая среда // Научные и технические библиотеки. 2005. № 9. С. 67-72.

7. Власова С.А. Реализация тематического поиска в электронном каталоге Библиотеки по естественным наукам Российской академии наук // Румянцевские чтения - 2018: Библиотеки и музеи как культурные и научные центры: историческая ретроспектива и взгляд в будущее : К 190-летию со времени основания Румянцевского музея : материалы Междунар. науч.-практ. конф. Москва, 2018. Ч. 1. С. $145-149$.

8. Власова С.А. Поисковые возможности сводного электронного каталога БЕН РАН / Чертковские чтения: Пространство культуры: книга, чтение, библиотека в культурной жизни России : сборник материалов четвертой науч.-практ. конф. (Москва, 4-5 декабря 2015 г.). Москва : Гос. публичная ист. б-ка России, 2016. С. 155-161.

9. Ивановский А.А. Возможности поиска по классификационным индексам в электронном каталоге: опыт Библиотеки по естественным наукам Российской академии наук // Петербургская библиотечная школа. 2016. № 4 (56). С. 64-69.

10. Ивановский А.А. Универсальная десятичная классификация как основа онтологии области знания: пример физиологии растений // Румянцевские чтения - 2019 : материалы Междунар. науч.практ. конф. (Москва, 23-24 апреля 2019 г.). Москва, 2019. Ч. 1. С. 329-332.

11. Якшин М.М., Каленов Н.Е. Классификаторы: создание базы данных терминологических словарей // Информационное обеспечение науки: новые технологии : сборник науч. тр. Москва : БЕН РАН, 2015. С. 137-146. 
12. Каленов Н.Е., Белоозеров В.Н. Формирование терминологических словарей по лексике классификационных систем // Научно-техническая информация. Сер. 1 : Организация и методика информационной работы. 2015. № 3. С. $60-69$.

13. Сысоев А.Н. Эмерджентность как критерий качества феномена классификации [Электронный ресурс] // Культура: теория и практика : электронный научный журнал. 2016. № 5-6 (14-15). URL: http://theoryofculture.ru/issues/69/892 (дата обращения: 15.04.2020).

14. Тютюнова В.С., Сысоев А.Н. Использование лингвистического метода при анализе «научного ландшафта» на основании данных каталога Библиотеки по естественным наукам РАН / Р Рмянцевские чтения - 2018 : Библиотеки и музеи как культурные и научные центры: историческая ретроспектива и взгляд в будущее : К 190-летию со времени основания Румянцевского музея : материалы Междунар. науч.-практ. конф. Москва, 2018. Ч. 3. С. 174-177.

15. Тютчева Н.И. Тематический поиск в ЭК: современное состояние и перспективы развития [Электронный ресурс] // Фонды и каталоги Кузбасса. Опыт. Проблемы. Решения : науч.-практ. сборник [2003]. Вып. 3. URL: http://old.kemrsl. $\mathrm{ru} /$ documents/founds/vip3/vip3.8.htm (дата обращения: 15.04.2020).
16. Скарук Г.А. Иерархические классификации в автоматизированном поиске // Труды ГПНТБ CO РАН. 2015. № 8. С. 267-274.

17. Скарук Г.А. Возможности поиска по классификационным индексам в электронном каталоге // Научные и технические библиотеки. 2016. № 3. C. 19-29. DOI: 10.33186/1027-3689-2016-3-19-29.

18. Лавренова О.А. Электронные каталоги: тенденции и практика РГБ // Научные и технические библиотеки. 2000. № 2. С. 29-35.

19. Лавренова О.А. Тематический поиск в электронных библиотеках: иллюзии и реальность [Электронный ресурс] / / Библиотеки и информационные ресурсы в современном мире науки, культуры, образования и бизнеса : XV Юбилейная междунар. конф. «Крым-2008». Москва : ГПНТБ России, 2008. URL: http://gpntb.ru/win/inter-events/crimea2008/ disk/133.pdf (дата обращения: 15.04.2020).

20. Лавренова О.А. Тематический поиск электронных ресурсов на основе классификационной модели организации знаний // Университетская книга. 2017. № 7. С. $58-61$.

21. Лавренова О.А. Задача интеграции технологий тематического поиска библиотечных ресурсов на основе словарей связанных данных (LOD vocabularies) // Румянцевские чтения - 2019 : материалы Междунар. науч.-практ. конф. (Москва, 23-24 апреля 2019 г.). Москва : Пашков дом, 2019. Ч. 2. С. 104-109.

\title{
Problems of Subject Retrieval in the Electronic Catalogue of Scientific Library: Review of Studies
}

\author{
Elena V. Dovbnya, \\ Library for Natural Sciences of the Russian Academy of Sciences, Central Library of Pushchino Scientific \\ Center of RAS, 3 Institutskaya Str., Pushchino, Moscow Region, 142290, Russia \\ ORCID 0000-0003-2222-8340; SPIN 8009-8721 \\ E-mail:pusikat13@rambler.ru
}

\begin{abstract}
Scientific libraries use different approaches and tools for subject retrieval. Issues related to electronic subject retrieval are widely discussed in the library community. The purpose of this review is to analyse publications of recent years on the aspects and problems related to subject retrieval in electronic catalogue (EC). For its preparation, the author used publications (and references to them) taken from the national bibliographic database - the Russian Science Citation Index (RSCI); the time coverage is 2003-2019. Particular attention in the studies is focused on the need to combine different information
\end{abstract}


retrieval languages (IRL), thesauruses, subject headings and keywords to ensure effective and multi-aspect search. Since the domestic libraries have accumulated a lot of experience in indexing and retrieval through hierarchical classifications (UDC and LBC), it is necessary to use this at the full extent in the electronic subject retrieval. The authors of the recent studies on subject retrieval consider being the most effective the functioning of classification systems as part of the complex of linguistic tools of electronic catalogue. In the electronic environment, it becomes possible to use classification indexes as a set of characters that maximally reflect the content of the document, what involves the use of all fragments of the index as independent retrieval elements. However, the lexical composition of the classification information retrieval languages needs to be modified for effective use in the electronic catalogue. The main directions here can be as follows: unification of the structure and vocabulary of classification schedules, optimization of the structure of classification systems (using facetization principles), as well as formalization of the use of grammar tools, (it is necessary to ensure the unambiguity of their interpretation in the electronic catalogue). The review presents and analyses the areas where the subject retrieval has been developed in the electronic environment for over the past 16 years. For example, there has been developed and is being implemented the superstructure over Universal Decimal Classification (Library for Natural Sciences of the Russian Academy of Sciences), which allows users of the library to remotely conduct full-fledged subject retrieval. The paper also presents the new developments by the Russian State Library on subject retrieval, in particular, the project "Presentation of classification metadata of electronic libraries using linked data technology". The author concludes that it is necessary to form a common space of related open data of various libraries.

Key words: library and information services, subject retrieval, electronic catalogue, hierarchical classifications, subject indexing, information retrieval languages, Library Bibliographic Classification, Universal Decimal Classification, scientific libraries.

Citation: Dovbnya E.V. Problems of Subject Retrieval in the Electronic Catalogue of Scientific Library: Review of Studies, Bibliotekovedenie [Russian Journal of Library Science], 2020, vol. 69, no. 4, pp. 367-374. DOI: 10.25281/0869-608X-2020-69-4-367-374.

\section{References}

1. Sukiasyan E.R. Homo Quaerens (The Seeking Man): On the Problem of Development of the Reader's Cognitive Capacities in the Searching Process, Nauchnye i tekhnicheskie biblioteki [Scientific and Technical Libraries], 2002, no. 4, pp. 7378 (in Russ.).

2. Levin G.L. Bibliographic Search: Theoretical Aspect, Bibliotekovedenie [Russian Journal of Library Science], 2000, no. 4, pp. 47-50 (in Russ.).

3. Lavrenova O.A. Subject Search in Digital Catalogues and Libraries, Bibliotekovedenie [Russian Journal of Library Science], 2004, no. 5, pp. 42-50 (in Russ.).

4. Skaruk G.A., Zharikova L.A., Stukalova A.A. Poiskovye yazyki elektronnykh katalogov: konspekt lektsii po kursu "Spravochno-poiskovyi apparat" [Search Languages of Electronic Catalogues: Lecture Notes on the Course "Reference and Search Apparatus"]. Novosibirsk, GPNTB SO RAN Publ., 2009, 44 p. (in Russ.).

5. Miniyarova Z.M. Approaches to Indexing in Electronic Catalogues. Element-By-Element Search Capabilities, Nauchnye i tekhnicheskie biblioteki [Scientific and Technical Libraries], 2005, no. 12, pp. 25-35 (in Russ.).

6. Miniyarova Z.M. Electronic Catalogue - A Specific Search Environment, Nauchnye i tekhnicheskie biblioteki [Scientific and Technical Libraries], 2005, no. 9, pp. 67-72 (in Russ.).

7. Vlasova S.A. The Implementation of Subject Search in the Electronic Catalogue of the Library for Natural Sciences of the Russian Academy of Sciences, $R u$ myantsevskie chteniya - 2018: Biblioteki i muzei kak kul'turnye inauchnye tsentry: istoricheskay a retrospektiva i vzglyad v budushchee: K 190-letiyu so vremeni osnovaniya Rumyantsevskogo muzeya: materialy Mezhdunar. nauch.-prakt. konf. [Proc. of the Int. Sci.-Pract. Conf. "Rumyantsev Readings - 2018: Libraries and Museums as Cultural and Scientific Centers: A Historical Retrospective and a Look into the Future: To the 190th Anniversary of the Foundation of the Rumyantsev $\mathrm{Mu}$ seum"]. Moscow, 2018, part 1, pp. 145-149 (in Russ.).

8. Vlasova S.A. Search Capabilities of the Union Electronic Catalogue of the Library for Natural Sciences of the Russian Academy of Sciences, Chertkovskie chteniya: Prostranstvo kul'tury: kniga, chtenie, biblioteka v kul'turnoi zhizni Rossii: sbornik materialov chetvertoi nauch.-prakt. konf. (Moskva, 4-5 dekabrya 2015 g.) [Proc. of the Fourth Sci.-Pract. Conf. "Chert- 
kov Readings: The Space of Culture: Book, Reading, Library in the Cultural Life of Russia” (Moscow, December 4-5, 2015)]. Moscow, Gosudarstvennaya Publichnaya Istoricheskaya Biblioteka Rossii Publ., 2016, pp. 155-161 (in Russ.).

9. Ivanovsky A.A. Capabilities of Searching by Classification Indexes in an Electronic Catalogue: An Experience of the Library for Natural Sciences of the Russian Academy of Sciences, Peterburgskaya bibliotechnaya shkola [Petersburg Library School], 2016, no. 4 (56), pp. 64-69 (in Russ.).

10. Ivanovsky A.A. Universal Decimal Classification as a Basis for Ontology of Knowledge Area: A Case Study on Plant Physiology, Rumyantsevskie chteniya - 2019: materialy Mezhdunar. nauch.-prakt. konf. (Moskva, 23-24 aprelya 2019 g.) [Proc. of the Int. Sci.-Pract. Conf. "Rumyantsev Readings - 2019" (Moscow, April 23-24, 2019)]. Moscow, 2019, part 1, pp. 329-332 (in Russ.).

11. Yakshin M.M., Kalenov N.E. Classifiers: Creating a Database of Terminology Dictionaries, Informatsionnoe obespechenie nauki: novye tekhnologii: sbornik nauch. tr. [Information Support of Science: New Technologies: collected scientific papers]. Moscow, BEN RAN Publ., 2015, pp. 137-146 (in Russ.).

12. Kalenov N.E., Beloozerov V.N. Developing of Terminological Dictionaries for Classification Systems Wordings, Nauchno-tekhnicheskaya informatsiya. Ser. 1: Organizatsiya i metodika informatsionnoi raboty [Scientific and Technical Information. Series 1: Organization and Methods of Information Work], 2015, no. 3, pp. 60-69 (in Russ.).

13. Sysoev A.N. Emergence as a Quality Criterion of the Phenomenon of Classification, Kul'tura: teoriya i praktika: elektronnyi nauchnyi zhurnal [Culture: Theory and Practice: electronic scientific journal], 2016, no. 5-6 (14-15). Available at: http://theoryofculture. $\mathrm{ru} /$ issues/69/892 (accessed 15.04.2020) (in Russ.).

14. Tyutyunova V.S., Sysoev A.N. Using the Linguistic Method in the "Scientific Landscape" Analysis Based on Data from the Catalogue of the Library for Natural Sciences of the Russian Academy of Sciences, Rumyantsevskie chteniya - 2018: Biblioteki i muzei kak kul'turnye i nauchnye tsentry: istoricheskaya retrospektiva i vzglyad v budushchee: K 190-letiyu so vremeni osnovaniya Rumyantsevskogo muzeya: materialy Mezhdunar. nauch.-prakt. konf. [Proc. of the Int. Sci.-Pract. Conf. "Rumyantsev Readings - 2018: Libraries and Museums as Cultural and Scientific
Centers: A Historical Retrospective and a Look into the Future: To the 190th Anniversary of the Foundation of the Rumyantsev Museum”]. Moscow, 2018, part 3, pp. 174-177 (in Russ.).

15. Tyutcheva N.I. Subject Search in Electronic Catalogue: Current State and Development Prospects, Fondy i katalogi Kuzbassa. Opyt. Problemy. Resheniya: nauch.-prakt. sbornik [Collections and Catalogues of Kuzbass. Experience. Problems. Solutions: scientific and practical collection], issue 3. Available at: http:// old.kemrsl.ru/documents/founds/vip3/vip3.8.htm (accessed 15.04.2020) (in Russ.).

16. Skaruk G.A. Hierarchical Classifications in Automated Search, Trudy GPNTB SO RAN [Proceedings of the SPSTL SB RAS], 2015, no. 8, pp. 267-274 (in Russ.).

17. Skaruk G.A. Capabilities of Searching by Classification Indexes in an Electronic Catalogue, Nauchnye i tekhnicheskie biblioteki [Scientific and Technical Libraries], 2016, no. 3, pp. 19-29. DOI: 10.33186/1027-3689-2016-3-19-29 (in Russ.).

18. Lavrenova O.A. Electronic Catalogues: Trends and Practices of the Russian State Library, Nauchnye i tekhnicheskie biblioteki [Scientific and Technical Libraries], 2000, no. 2, pp. 29-35 (in Russ.).

19. Lavrenova O.A. Subject Search in Electronic Libraries: Illusions and Reality, Biblioteki i informatsionnye resursy $v$ sovremennom mire nauki, kul'tury, obrazovaniya i biznesa: XV Yubileinaya mezhdunar. konf. "Krym-2008” [Libraries and Information Resources in the Modern World of Science, Culture, Education and Business: The 15th Anniversary International Conference “Crimea-2008”]. Moscow, GPNTB Rossii Publ., 2008, Available at: http://gpntb.ru/win/ inter-events/crimea2008/disk/133.pdf (accessed 15.04.2020) (in Russ.).

20. Lavrenova O.A. The Subject Search for Electronic Resources Based on the Classification Model of Knowledge Organization, Universitetskaya kniga [University Book], 2017, no. 7, pp. 58-61 (in Russ.).

21. Lavrenova O.A. The Technology Integration for Subject Search of Library Resources Based on LOD Vocabularies, Rumyantsevskie chteniya - 2019: materialy Mezhdunar. nauch.-prakt. konf. (Moskva, 23-24 aprelya 2019 g.) [Proc. of the Int. Sci.-Pract. Conf. "Rumyantsev Readings - 2019" (Moscow, April 23-24, 2019)]. Moscow, Pashkov Dom Publ., 2019, part 2, pp. 104-109 (in Russ.). 